\title{
Beneficio económico en la utilización de reserva fraccional de flujo en lesiones coronarias intermedias y su impacto clínico a un año
}

\author{
Economic benefits of fractional flow reserve utilization on intermediate lesions and its \\ clinical impact after one year-follow up
}

\author{
Horacio A. Medina-de Chazal*, Fernando Cohen, Franco Pallavicini, Alejandro D. Fernández, \\ Carla R. Agatiello y Daniel H. Berrocal \\ Servicio de Hemodinamia y Cardiología Intervencionista. Hospital Italiano, Buenos Aires, Argentina
}

\begin{abstract}
Resumen
Antecedentes: La reserva de flujo fraccional (FFR) es una herramienta con evidencia demostrada para guiar las angioplastias coronarias. El reembolso por los sistemas de cobertura de salud es parcial o nulo a pesar de frecuentemente diferir la angioplastia. Nuestro objetivo fue determinar el beneficio económico de la utilización del FFR en la evaluación de lesiones intermedias, y evaluar asimismo puntos finales clínicos en el seguimiento a un año. Métodos: Estudio observacional prospectivo que incluyó una cohorte de pacientes consecutivos con lesiones coronarias intermedias, evaluadas con FFR, entre abril de 2013 y marzo de 2016. Para el análisis económico se evaluaron los recursos específicos utilizados para la realización del procedimiento. Se analizaron puntos finales clínicos (muerte cardiovascular, revascularización de la arteria objetivo e infarto agudo de miocardio) durante la internación y en el seguimiento a un año. Resultados: Se incluyeron 222 lesiones en 151 pacientes consecutivos. Se registró FFR positivo en el $26.1 \%$ de las lesiones evaluadas. Se estimó que sin la utilización de FFR, 126 pacientes hubieran sido tratados con angioplastia transluminal coronaria y 25 con cirugía de revascularización miocárdica. El costo estimado con la utilización de FFR fue US\$ 891,290.08, mientras que sin el mismo hubiera sido de US\$1,557,352. Esto implicó un ahorro del 43\% de los gastos. Se observaron una muerte de origen cardiovascular y dos reinternaciones en el grupo FFR positivo en el seguimiento a un año. Conclusiones: La revascularización de lesiones intermedias guiada por FFR resultó en un beneficio económico al reducir los costos generales sin resultar clínicamente perjudicial.
\end{abstract}

Palabras claves: Enfermedad coronaria. Intervenciones coronarias. Lesiones coronarias intermedias. Fracción de reserva de flujo coronario. Costo beneficio. Argentina.

\begin{abstract}
Background: Fractional flow reserve (FFR) is a proven technology for guiding percutaneous coronary intervention, but it is not reimbursed despite the fact that it frequently allows to defer revascularization. Our goal was to determine the economic
\end{abstract}

\section{Correspondencia:}

*Horacio A. Medina de Chazal

Tte. Gral. Juan Domingo Perón 4190, C.A.B.A

Fecha de recepción: 14-11-2018

Fecha de aceptación: 10-12-2018

C1199ABB, Buenos Aires, Argentina

E-mail: horacio.medina@ hospitalitaliano.org.ar

DOI: 10.24875/ACM.M19000037
Disponible en internet: 09-12-2019 Arch Cardiol Mex. 2019;89(4):308-314 www.archivoscardiologia.com 1405-9940/@ 2019 Instituto Nacional de Cardiología Ignacio Chávez. Publicado por Permanyer. Este es un artículo open access bajo la licencia CC BY-NC-ND (http://creativecommons.org/licenses/by-nc-nd/4.0/) 
benefit of FFR on intermediate lesions, as well as the clinical endpoints at 1 year follow up. Methods: Observational prospective study that included consecutive patients with intermediate lesions evaluated with FFR between April 2013 and March 2016. For the economic analysis we evaluated the specific resources used during the procedure. Clinical endpoints including cardiovascular death, target lesion revascularization and acute myocardial infarction, were followed up over a one-year period. Results: FFR was performed on 222 lesions in 151 consecutive patients. FFR was positive in $26.1 \%$ of the assessed lesions. The estimated total cost using FFR was US\$ 891,290.08 while cost estimate without FFR was US\$1,557,352, meaning 43\% in cost savings. There was one cardiovascular death and two readmissions during follow up in the positive FFR group. Conclusions: FFR guided revascularization on intermediate coronary lesions resulted in an economic benefit by reducing overall costs without harming clinical outcomes.

Key words: Coronary disease. Coronary interventions. Intermediate coronary lesions. Fractional flow reserve. Cost benefit. Argentina.

\section{Introducción}

La enfermedad coronaria continúa siendo la primera causa de muerte en todo el mundo ${ }^{1}$. Argentina no es la excepción: la enfermedad cardiovascular se mantiene como la principal causa de muerte, y representa el $32 \%$ del total ${ }^{2}$. La revascularización miocárdica es el tratamiento de elección, tanto quirúrgica como percutánea, con una mayor tasa de utilización en países occidentales. Solo en EE.UU. se realizan más de 1 millón de intervenciones coronarias percutáneas o procedimientos de revascularización en forma anual ${ }^{3}$.

En Argentina, según un relevamiento realizado en el año 2011 por la División Salud de la Organización para la Cooperación y el Desarrollo Económico, se realizan 942 angioplastias transluminales coronarias (ATC) por millón de habitantes en forma anual, lo que equivale a aproximadamente $38 \mathrm{mil}$ angioplastias anuales, cifra que, si bien se encuentra alejada de los países desarrollados, va en aumento.

La revascularización mediante ATC o mediante cirugía de revascularización miocárdica (CRM) tiene un importante papel dentro del tratamiento de estos pacientes, pero ambas pueden tener un efecto paradójico. En presencia de isquemia miocárdica, la revascularización reduce los síntomas ${ }^{4,5}$ y mejora el pronóstico ${ }^{6-8}$, pero en su ausencia la revascularización empeora los resultados ${ }^{9}$.

La cinecoronariografía (CCG) es el patrón de oro para el diagnóstico de enfermedad coronaria en los vasos coronarios epicárdicos, aunque la cuantificación exacta de una lesión puede estar limitada por diferentes motivos. Una de las principales limitaciones es la dificultad para determinar el diámetro real del vaso de referencia, a la hora de comparar con la lesión en estudio ${ }^{10}$. Si el mismo estuviese enfermo, como ocurre en pacientes que presentan enfermedad coronaria difusa, el grado de estenosis producido por la lesión en estudio podría infraestimarse. Así también, la presencia de artefactos del contraste por turbulencia, acortamientos de algunos segmentos en diferentes proyecciones, calcificaciones, lesiones de localización ostial o bifurcaciones puede contribuir a la inexactitud de la evaluación ${ }^{11}$. Sumado a esto, existe una considerable variabilidad interobservador en el momento de la evaluación ${ }^{12}$.

En Argentina, como en la mayoría de los lugares, la decisión de revascularizar es principalmente tomada basándose en la apariencia angiográfica de las lesiones coronarias ${ }^{13}$, frecuentemente desconociendo si las mismas son realmente causantes de isquemia miocárdica: solo el $30 \%$ de los pacientes se presentan a la evaluación angiográfica con una prueba de apremio con evidencia de isquemia miocárdica que justifique sus síntomas ${ }^{14,15}$. Aun disponiendo de dichas pruebas, estas presentan diversas falencias. La prueba ergométrica graduada (prueba más frecuentemente utilizada) tiene precisión inadecuada en diversos pacientes ${ }^{16}$; el ecocardiograma de estrés y la SPECT (pruebas con mayor precisión para la identificación de isquemia) presentan capacidad limitada para definir la localización de la isquemia ${ }^{17}$, además de ser costosas y de acotada disponibilidad. Como consecuencia, muchas lesiones coronarias que no son hemodinámicamente significativas son innecesariamente sometidas a ATC o CRM, con un costo considerable para el sistema de salud y en detrimento del paciente.

Como es conocido, la medición de la reserva de flujo fraccional (FFR) permite, de forma simple, rápida y reproducible, la identificación de lesiones coronarias funcionalmente significativas, con especial beneficio en las lesiones coronarias intermedias (estenosis entre 
50-69\%). Estudios previos mostraron que solo el 35\% de las mismas presentan un FFR que indica la presencia de isquemia ${ }^{18}$.

Existe abundante evidencia proveniente de estudios aleatorizados para demostrar el beneficio clínico y económico de la utilización del FFR en comparación con la revascularización guiada solo por angiografía.

El estudio DEFER ${ }^{19}$ demostró que no existe beneficio en la angioplastia de lesiones hemodinámicamente no significativas determinado mediante medición de FFR. El estudio FAME $^{9}$ mostró beneficios significativos en términos de morbilidad y mortalidad en la revasularización de lesiones coronarias guiada por FFR.

La estrategia de revascularización guiada por FFR demostró además reducción en los $\operatorname{costos}^{9,20}$, lo que prodría significar un potencial beneficio económico para el sistema de salud.

Los sistemas para medición de FFR han estado disponible en Argentina desde hace casi diez años, sin embargo, es un método subutilizado en la práctica diaria. La cobertura de dicho procedimiento por parte de las obras sociales y prepagas es limitada, por lo que, a pesar de su potencial ahorro en términos económicos, la realidad actual atenta contra su utilización.

Teniendo en cuenta el número y la complejidad creciente de las intervenciones coronarias en Argentina, el potencial ahorro económico mediante la implementación de FFR para guiar la revascularización en lesiones coronarias intermedias, en comparación a la evaluación angiográfica, podría ser relevante para el sistema de salud.

El objetivo de este estudio fue determinar el beneficio económico de la utilización de FFR en la evaluación de lesiones intermedias y su impacto clínico en el seguimiento a un año.

\section{Materiales y métodos}

Se realizó un estudio observacional prospectivo que incluyó una cohorte de pacientes consecutivos que presentaron lesiones coronarias intermedias en la angiografía, evaluadas con FFR, entre abril de 2013 y marzo de 2016. Se definió como lesión coronaria intermedia o moderada aquella estenosis luminal entre 50 y $69 \%$ evaluada por angiografía. Se tomó como FFR positivo a aquel valor menor de $0.80^{\circ}$, luego de obtenida máxima hiperemia mediante la infusión intracoronaria de adenosina. Se evaluaron las características clínicas de la población en estudio y se analizaron puntos finales clínicos y económicos durante la internación y en el seguimiento al año.
Para el análisis económico se evaluaron los recursos específicos utilizados para la realización del procedimiento. Los costos de la ATC se calcularon teniendo en cuenta la totalidad de recursos utilizados tanto diagnósticos como terapéuticos, considerando el valor en el mercado argentino en dólares, para evitar los ajustes por oscilaciones en la moneda local durante el periodo analizado. El costo de la CRM fue considerado de acuerdo al valor de un módulo preestablecido que incluye la totalidad de los recursos necesarios, incluida la internación en unidad coronaria durante cinco días.

Se revisaron las estadísticas del servicio en el periodo evaluado, y se definió una tasa de utilización de 1.78 stents por paciente ( 0.51 stents convencionales y 1.27 stents liberadores de fármaco) y una media de 1.1 días de internación en la unidad coronaria por paciente sometido a angioplastia coronaria.

Se analizaron asimismo los puntos finales: muerte cardiovascular, revascularización de la arteria objetivo e infarto agudo de miocardio, durante la internación y en el seguimiento al año.

\section{Análisis estadístico}

Las variables categóricas se reportaron como frecuencias, mientras que las continuas fueron descriptas por su media y desviación estándar o mediana y rango intercuartilo.

\section{Resultados}

Se analizaron 222 lesiones intermedias en los primeros 151 pacientes consecutivos a quienes se realizó FFR en el periodo comprendido entre abril de 2013 y marzo de 2016. Las características de la población en estudio se resumen en la tabla 1. De los pacientes incluidos, solo el $42 \%$ poseían prueba de apremio positiva, previo al estudio angiográfico, siendo el SPECT el estudio más solicitado (73.4\%). El síndrome coronario agudo sin elevación del ST fue el cuadro clínico que más frecuentemente motivo la realización de CCG (40\%) (Fig. 1). El $67.3 \%$ de los estudios se realizó en pacientes internados.

El 26.1\% de las lesiones evaluadas arrojaron un valor menor de 0.80 (58 lesiones en 33 pacientes), siendo la arteria descendente anterior la más estudiada (49.74\%) (Tabla 2).

De los 151 pacientes analizados, 53 recibieron tratamiento percutáneo, 12 quirúrgico y 86 tratamiento médico. De no haberse utilizado FFR en la evaluación de 
Tabla 1. Características de la población $(n=151)$

\begin{tabular}{|c|c|}
\hline Variable & Valor (\%) \\
\hline Edad (media \pm DS) & $69.22 \pm 11.1$ años \\
\hline Sexo masculino & $92(60.92 \%)$ \\
\hline Tabaquismo actual & $21(14 \%)$ \\
\hline Diabetes & $39(26 \%)$ \\
\hline Hipertensión arterial & $111(74 \%)$ \\
\hline Dislipemia & $93(62 \%)$ \\
\hline $\begin{array}{l}\text { Enfermedad coronaria } \\
\text { conocida }\end{array}$ & $68(45.3 \%)$ \\
\hline $\begin{array}{l}\text { Enfermedad vascular } \\
\text { extracoronaria }\end{array}$ & $32(21.3 \%)$ \\
\hline ATC previa & $50(33.3 \%)$ \\
\hline CRM previa & $7(4.6 \%)$ \\
\hline $\begin{array}{l}\text { Cirugía cardíaca previa } \\
\text { distinta a CRM }\end{array}$ & $4(2.6 \%)$ \\
\hline Anticoagulación oral previa & $11(7.3 \%)$ \\
\hline Internado & $101(67.3 \%)$ \\
\hline Apremio positivo & $\begin{array}{c}64 \text { (42\%) (SPECT } 47[73.4 \%] \text {, eco } \\
\text { de estrés } 5[7.8 \%], \text { angio-TC } \\
6[9,3 \%], \text { PEG } 6[9,3 \%])\end{array}$ \\
\hline
\end{tabular}

ATC: angioplastia transluminal coronaria; CRM: cirugía de revascularización miocárdica; PEG: prueba ergométrica graduada; SPECT: single photon emission computed tomography.

Tabla 2. Distribución de las arterias estudiadas ( $n=199)$

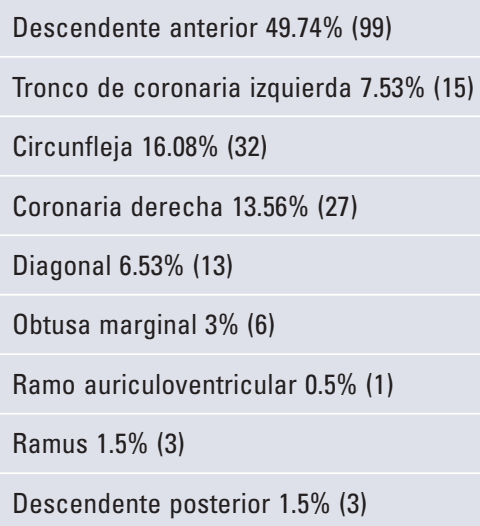

las lesiones intermedias, 126 pacientes hubiesen sido sometidos a angioplastia y 25 a CRM (Fig. 2). La medición de FFR en lesiones intermedias generó un ahorro de 141 stents (100.61 DES y 40.39 BMS teniendo en cuenta la tasa de utilización de stents en el servicio)

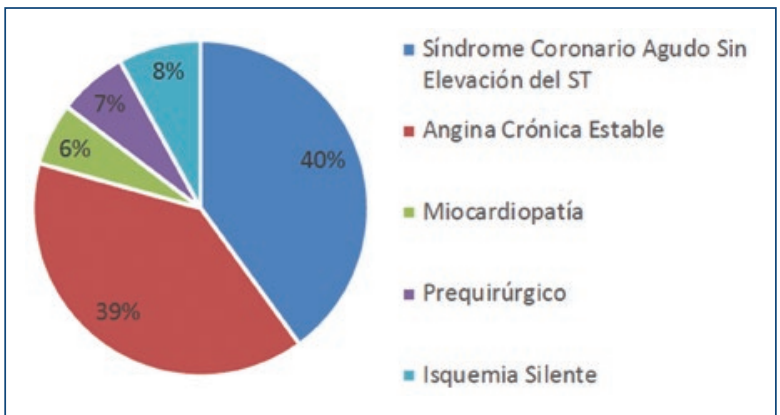

Figura 1. Diagnóstico que motivó el estudio de la anatomía coronaria. ACE: angina crónica estable; SCASEST: síndrome coronario agudo sin elevación del ST.

y de 98.8 días de internación en unidad coronaria. En términos económicos, de no haberse utilizado la medición de este índice funcional, el costo total hubiese sido de US $\$ 1,557,352$. Con la medición de FFR, el importe total fue de US\$ 891,290.08, lo que representó un ahorro de US\$666,061.92, o, expresado en términos relativos, del $42.77 \%$ (Fig. 3).

Con respecto a eventos clínicos, se registró una sola muerte cardiovascular durante la internación en el grupo FFR positivo (paciente con trombo en el ventrículo izquierdo, fallecido por accidente cerebrovascular a la espera de CRM). Tres pacientes tuvieron reinternaciones por angina inestable en el seguimiento, dos de ellos en el grupo que recibió angioplastia y uno en el grupo de tratamiento médico. Solo uno requirió nueva angioplastia por reestenosis del stent implantado en el evento índice. En 16 pacientes se perdió el seguimiento luego de la realización de la angiografía coronaria.

De los pacientes analizados, solo dos presentaron complicaciones graves relacionadas con el procedimiento (sangrado del sitio de punción con requerimiento de transfusión y disección coronaria durante la angioplastia que requirió CRM de urgencia).

\section{Discusión}

Los resultados del presente estudio demuestran un ahorro económico en la revascularización guiada por FFR comparado con la angiografía estándar a expensas de la reducción de la tasa de revascularización, tanto percutánea como quirúrgica. Esto no se tradujo en perjuicio clínico en la internación y en el seguimiento al año. Si bien se registró una muerte en uno de los grupos, esta no fue causada por el procedimiento 


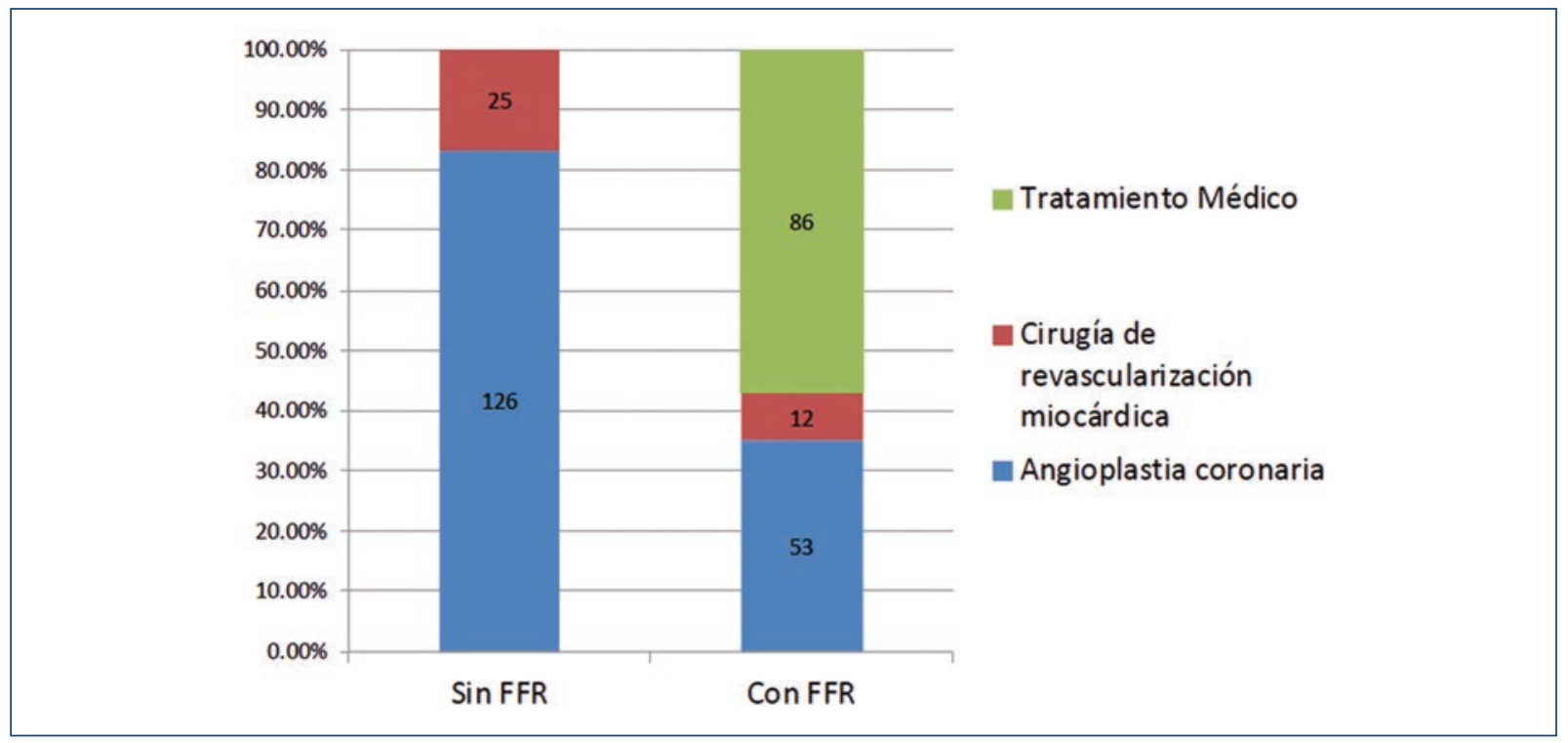

Figura 2. Diferencias entre los tratamientos propuestos sin la medición de la FFR y después de su medición. ATC: angioplastia transluminal coronaria; CRM: cirugía de revascularización miocárdica; FFR: reserva de flujo fraccional.

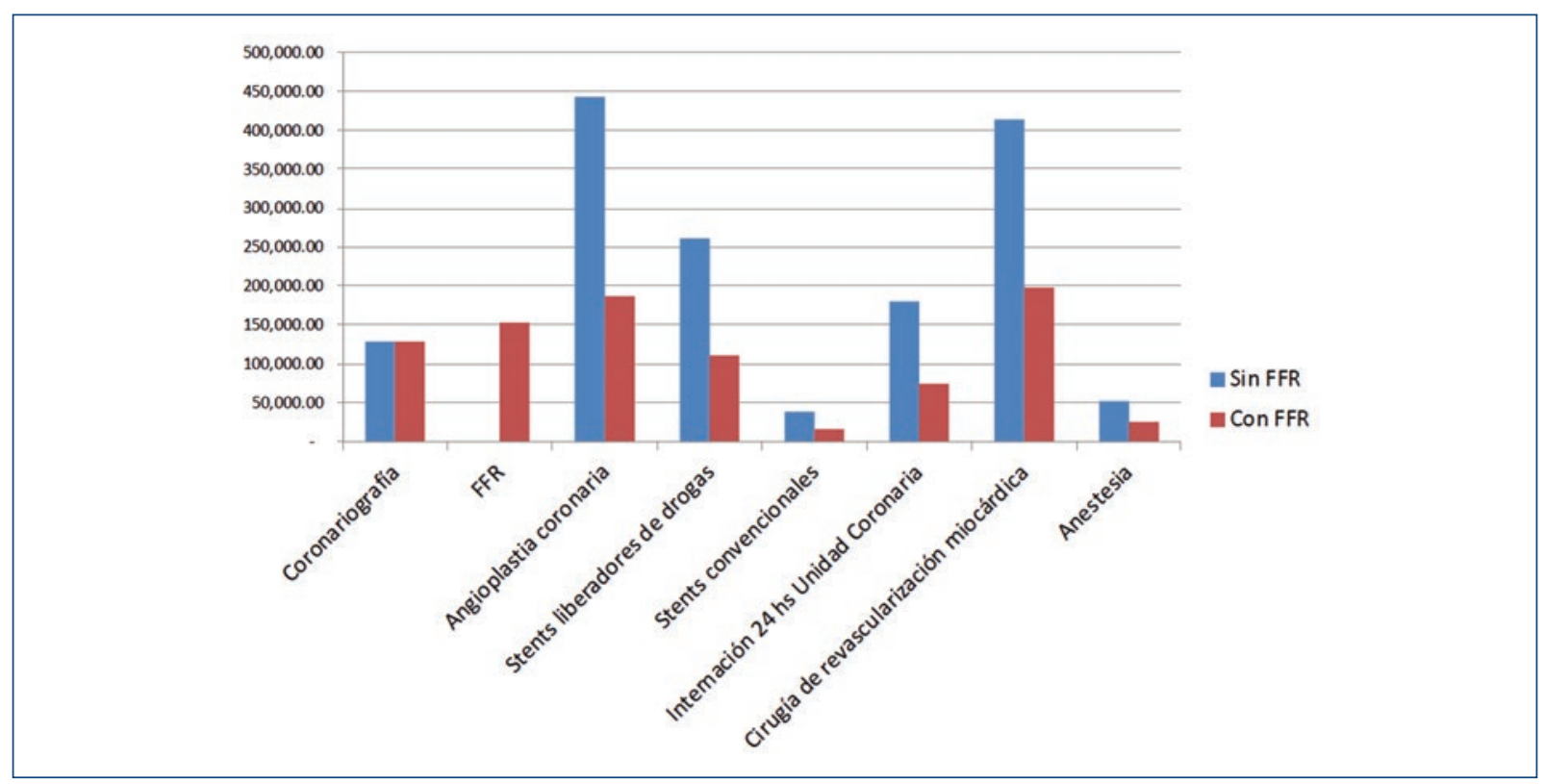

Figura 3. Diferencia en costos (expresado en dólares americanos) de acuerdo con la utilización de FFR. ATC: angioplastia transluminal coronaria; BMS: bare metal stent, CCG: cinecoronariografía; CRM: cirugía de revascularización miocárdica; DES: drug eluting stent, FFR: fracción de reserva de flujo fraccional.

diagnóstico o terapéutico, sino que se vincula con el azar.

Como fue mencionado previamente, la angiografía, a pesar de ser el estándar de referencia en la evaluación de lesiones intermedias, es un mal predictor de lesiones generadoras de isquemia. En el presente estudio se evidenció un valor de FFR $>0.80$ en el $73.9 \%$ de las lesiones estudiadas, coincidente con lo reportado en la literatura. En el estudio $\mathrm{FAME}^{9}$, el $65 \%$ de las lesiones intermedias basadas en la estimación 
visual presentaron FFR $>0.80$ y un $20 \%$ de las estenosis, entre 70 y $90 \%$, no fueron responsables de un FFR $<0.80$.

Frecuentemente la apariencia angiográfica de las lesiones que producen isquemia es idéntica a las que no lo hacen, esto es más marcado en las lesiones moderadas $^{19}$. Demostrar la presencia de isquemia resulta crucial, ya que representa el predictor más importante de eventos adversos en el seguimiento alejado ${ }^{8}$. Así también, se ha documentado que la angioplastia sobre lesiones no productoras de isquemia no resulta beneficiosa, e incluso puede ser perjudicial en términos de complicaciones periprocedimiento y en el seguimiento alejado, con el consecuente incremento de los costos $^{18}$. La utilización de FFR en este contexto optimiza los resultados clínicos, con disminución tanto de los riesgos inherentes a los procedimientos como de los costos.

A pesar de la robusta evidencia de los beneficios de la angioplastia guiada por FFR, dicha estrategia es utilizada en Argentina en un porcentaje relativamente bajo, una de sus principales razones son sus costos. Los recursos para la medición de FFR son cubiertos en forma parcial o nula por parte del sistema de salud, lo que agrega un costo no recuperable en el presupuesto de los diversos centros de cardiología intervencionista. En contraste, los sistemas de salud realizan pagos adicionales en términos de recursos para revascularización y de estadía hospitalaria que podrían evitarse.

Nuestro análisis económico tiene limitaciones que debemos mencionar. Representa un estudio unicéntrico, el número de pacientes incluidos en el estudio es relativamente bajo $\mathrm{y}$, al no ser aleatorizado, los procedimientos y costos del grupo sin FFR se obtuvieron de un cálculo efectuado basado en las tazas de uso de procedimientos y dispositivos.

El número total tanto de pacientes como de lesiones intermedias que son evaluadas sin evidencia previa de isquemia en Argentina es desconocido. Por esta razón, resulta dificultoso estimar el potencial impacto económico total en la implementación de FFR en forma generalizada.

Nuestro análisis representa la evaluación económica durante la internación. Esto sería equivalente, según algunos reportes, al $90 \%$ del costo total por paciente ${ }^{20}$, aproximadamente. Algunos autores demostraron en el seguimiento al año, un ahorro que puede llegar al $30 \%$, entre los costos totales de ambas estrategias ${ }^{20}$. Esto significa un potencial ahorro adicional no evaluado en el presente trabajo.

\section{Conclusión}

La implementación rutinaria de una estrategia guiada por FFR en lesiones intermedias generó un importante cambio de conducta en términos de tratamiento, y en un grupo de pacientes que estaban planeados para revascularización determinó la implementación final de tratamiento médico en gran parte de ellos. Este cambio de conducta terapéutica supuso un claro beneficio económico sin generar perjuicios clínicos para los pacientes en el seguimiento a un año.

\section{Financiamiento}

No se recibió patrocinio de ningún tipo para llevar a cabo este estudio/artículo.

\section{Conflictos de intereses}

Los autores declaran no tener ningún conflicto de intereses.

\section{Responsabilidades éticas}

Protección de personas y animales. Los autores declaran que para esta investigación no se han realizado experimentos en seres humanos ni en animales.

Confidencialidad de los datos. Los autores declaran que en este artículo no aparecen datos de pacientes.

Derecho a la privacidad y consentimiento informado. Los autores declaran que en este artículo no aparecen datos de pacientes.

\section{Bibliografía}

1. Nabel EG, Braunwald E. A Tale of Coronary Artery Diasease and Myocardial Infarction. N Engl J Med. 2012;366:54-63.

2. Indicadores Básicos, 2005. Disponible en: http://www.deis.gov.ar/.

3. Rosamond, W, Flegal K, Friday G, Furie K, Go A, Greenlund K, et al. Heart disease and stroke statistics - 2007 Update: A report from the American Heart Association Statistics Committee and Stroke Statistics Subcommittee. Circulation. 2007;115(5):e69-171.

4. Pocock S. Coronary angioplasty versus medical therapy for angina: The second randomised intervention treatment of angina (RITA-2) trial. Lancet. 1997;350:461-8.

5. Fihn SD, Gardin JM, Abrams J, Berra K, Blankenship JC, Dallas AP, et al. 2012 ACCF/AHA/ACP/AATS/PCNA/SCAI/STS Guideline for the Diagnosis and Management of Patients With Stable Ischemic Heart Disease: A Report of the American College of Cardiology Foundation/American Heart Association Task Force on Practice Guidelines, and the American College of Physicians, American Association for Thoracic Surgery, Preventive Cardiovascular Nurses Association, Society for Cardiovascular Angiography and Interventions, and Society of Thoracic Surgeons. Circulation. 2012;126:e354-e471

6. Erne P, Schoenenberger AW, Burckhardt D, Zuber M, Kiowski W, Buser PT, et al. Effects of Percutaneous Coronary Interventions in Silent Ischemia. JAMA. 2007;297:1985-91.

7. Shaw, LJ, Berman DS, Maron DJ, Mancini GB, Hayes SW, Hartigan PM, et al. Optimal medical therapy with or without percutaneous coronary intervention to reduce ischemic burden: results from the Clinical Outcomes Utilizing Revascularization and Aggressive Drug Evaluation (COURAGE) trial nuclear substudy. Circulation. 2008;117:1283-91. 
8. Shaw LJ, Iskandrian AE. Prognostic value of gated myocardial perfusion SPECT. J Nucl. Cardiol. 2004;11:171-85.

9. Tonino P, De Bruyne B, Pijls N, Siebert U, Fumiaki I, van't Veer M. Fractional Flow Reserve versus Angiography for Guiding Percutaneous Coronary Intervention. N Engl J Med. 2009;360:213-24.

10. Topol E, Nissen S. Our Preoccupation With Coronary Luminology. Circulation. 1995;92: 2333-342.

11. Fischer JJ, Samady H, McPherson JA, Sarembock IJ, Powers ER, Gimple LW, et al. Comparison between visual assessment and quantitative angiography versus fractional flow reserve for native coronary narrowings of moderate severity. Am J Cardiol. 2002;90:210-5.

12. Zir L, Miller S, Dinsmore R, Gilbert J, Harthorne JW, et al. Interobserver variability in coronary angiography. Circulation 1976;627-32.

13. Boden W, O'Rourke R, Teo K, Hartigan P, Maron P, Kostuk W, et al. Optimal Medical Therapy with or without PCI for Stable Coronary Disease. N Engl J Med. 2007;356:1503-16

14. Topol EJ, Ellis SG, Cosgrove DM, Bates ER, Muller DW, Schork NJ, et al. Analysis of coronary angioplasty practice in the United States with an insurance-claims data base. Circulation. 1993;87:1489-97.
15. Kern MJ, Donohue TJ, Aguirre FV, Bach RG, Caracciolo EA, Wolford T, et al. Clinical outcome of deferring angioplasty in patients with normal translesional pressure-flow velocity measurements. J Am Coll Cardiol. 1995;25:178-87.

16. Chest pain of recent onset: assessment and diagnosis. Clin. Guidel. [CG95]

17. Lima RS, Watson DD, Goode AR, Siadaty MS, Ragosta M, Beller GA, et al. Incremental value of combined perfusion and function over perfusion alone by gated SPECT myocardial perfusion imaging for detection of severe three-vessel coronary artery disease. J Am Coll Cardiol. 2003;42:64-70.

18. Pijls NH, van Schaardenburgh P, Manoharan G, Boersma E, Bech JW, van't Veer M, et al. Percutaneous Coronary Intervention of Functionally Nonsignificant Stenosis. 5-Year Follow-Up of the DEFER Study. J Am Coll Cardiol. 2007;49:2105-11.

19. Bech GJ, De Bruyne B, Pijls NH, de Muinck ED, Hoorntje JC, Escaned J, et al. Fractional flow reserve to determine the appropriateness of angioplasty in moderate coronary stenosis: A randomized trial. Circulation. 2001;103:2928-34.

20. Fearon WF, Bornschein B, Tonino PA, Gothe RM, Bruyne BD, Pijls NH, et al. Economic evaluation of fractional flow reserve-guided percutaneous coronary intervention in patients with multivessel disease. Circulation. 2010;122:2545-50 